\title{
Clinical Cerenkov Luminescence Imaging of ${ }^{18}$ F-FDG
}

\author{
Daniel L.J. Thorek ${ }^{1}$, Christopher C. Riedl ${ }^{1}$, and Jan Grimm ${ }^{1,2}$ \\ ${ }^{I}$ Department of Radiology, Memorial Sloan-Kettering Cancer Center, New York, New York; and ${ }^{2}$ Program in Molecular \\ Pharmacology and Chemistry, Memorial Sloan-Kettering Cancer Center, New York, New York
}

The aim of this study was to determine the feasibility of Cerenkov luminescence $(\mathrm{CL})$ imaging of patients undergoing diagnostic ${ }^{18} \mathrm{~F}-$ FDG scans to detect nodal disease. Methods: Patients undergoing routine ${ }^{18} \mathrm{~F}-\mathrm{FDG} \mathrm{PET} / \mathrm{CT}$ for various malignancies consented to being scanned for CL. White-light and Cerenkov images (5-min acquisition) of the surface of the patient contralateral to and at the site of nodal ${ }^{18} \mathrm{~F}-\mathrm{FDG}$ uptake were acquired using a cooled, intensified charge-coupled-device camera. Results: The camera demonstrated linear correlation between activity and counts into the low nanocurie range using ${ }^{18} \mathrm{~F}-\mathrm{FDG}$. Imaging of patients revealed the presence of ${ }^{18} \mathrm{~F}-\mathrm{FDG}$ uptake in nodes that demonstrated uptake on PET. A correlation between maximum standardized uptake value from PET and counting rate per area on the CL imaging was established. Conclusion: CL imaging with diagnostic doses of ${ }^{18} \mathrm{~F}-\mathrm{FDG}$ is feasible and can aid in detecting disease in the clinical setting.

Key Words: Cerenkov luminescence imaging; ${ }^{18} \mathrm{~F}-\mathrm{FDG}$; PET/CT; clinical

J Nucl Med 2014; 55:95-98

DOI: 10.2967/jnumed.113.127266

$\mathbf{O}$

ptical imaging technologies, such as fluorescent, bioluminescent, and photoacoustic imaging, are powerful methods for the sensitive and high-resolution imaging of biomedical systems. In the basic science and preclinical settings, these techniques have delivered tremendous advances in understanding disease biology (1). They also have considerable utility in noninvasive evaluation of efficacy for drug development $(2,3)$. The capability of such technologies to detect pathologic molecular processes, particularly to identify malignancy, has been a major driving force in their development.

Recently, visible light emissions from microfluidic chips used for ${ }^{18} \mathrm{~F}$ radiochemistry were observed (4). This detection of Cerenkov radiation from a positron-emitting radionuclide commonly used in nuclear medicine was then quickly demonstrated in living mice $(5,6)$. This work has spurred considerable interest in the use of Cerenkov luminescence (CL) for optical imaging with radioactive sources. CL is optical wavelength photons produced by the charged particles emitted during radioactive decay traveling through a dielectric medium faster than the phase velocity of that medium (7).

Imaging of this light provides the potential to use clinical radiotracers for optical techniques, thus offering a unique opportunity to harness the strength of both nuclear and optical imaging using

\footnotetext{
Received Jun. 5, 2013; revision accepted Sep. 16, 2013.

For correspondence or reprints contact: Jan Grimm, 1275 York St., Box 248, Memorial Sloan-Kettering Cancer Center, New York, NY, 10065.

E-mail: grimmj@mskcc.org

Published online Sep. 27, 2013.

COPYRIGHT (C) 2014 by the Society of Nuclear Medicine and Molecular Imaging, Inc.
}

existing radiotracers as optical agents for high-throughput evaluation of tracers and drugs (8). For example, in small animals, $3^{\prime}$-deoxy$3^{\prime}-{ }^{18} \mathrm{~F}$-fluorothymidine and the approved ${ }^{18} \mathrm{~F}-\mathrm{FDG}$ have been used to evaluate an antiangiogenic drug in multiple disease models, showing strongly correlated CL and PET imaging results (9).

However, the levels of light produced by positron emitters are low, several orders of magnitude less than standard fluorescence imaging, which is again several orders less bright than ambient room light $(10,11)$, Overall, CL is about 9 orders of magnitude lower than room lighting. In addition, the intensity of the continuous spectrum of CL is inversely proportional to the square of the wavelength, weighting the emission toward the ultraviolet and blue range. These factors have been perceived as significant challenges for clinical translation. A CL image of a single hyperthyroid patient with a therapeutic dose of ${ }^{131} \mathrm{I}$ was recently published (12). Although no value for thyroid uptake was given, it can reasonably be assumed that approximately half the dose $(275 \mathrm{MBq})$ accumulated in the thyroid - still, a significantly high dose. Such levels can be accomplished with iodine uptake in the thyroid but not with other tracers used in nuclear medicine. We were interested in the possibility of using CL in the diagnostic imaging context of PET tracers, especially the ubiquitous ${ }^{18} \mathrm{~F}$-FDG. Specifically, our question was whether it is feasible to detect nodal disease using $\mathrm{CL}$ imaging in patients undergoing routine PET/CT scans. We here report a pilot study that was the first, to our knowledge, to demonstrate the clinical feasibility of CL imaging with the established PET tracer ${ }^{18} \mathrm{~F}$-FDG.

\section{MATERIALS AND METHODS}

\section{Acquisitions}

An intensified charge-coupled-device (CCD) camera (Mega10z; Stanford Photonics) was used to image CL. The single-photon-counting camera consists of a dual microchannel plate intensifier system behind a photocathode that is read off a Peltier-cooled CCD (XX285; Sony Corp.). The optics consisted of a quartz high-ultraviolet-transmission 50-mm $f / 0.8$ lens and a long-pass filter with a cutoff at $605 \mathrm{~nm}$ (Chroma Technology). The camera has an effective read noise of zero and was run at maximum gain for greatest sensitivity. High-frame-rate acquisitions (120 frames/s) were integrated in the Piper Control software (Stanford Photonics), a background baseline was subtracted, and the images were processed and analyzed in ImageJ (National Institutes of Health) (13).

In vitro measurements were made using the camera mounted on a custom-built light-tight box (Stanford Photonics). For patient imaging, the camera with lens and filter was mounted on a standard photography tripod, and the subjects were imaged at a distance of approximately $8 \mathrm{~cm}$. The patients were imaged with the room lights on for a white-light photograph and then were imaged with the lights off, the light-sealed door closed, and an optical drape used to eliminate any remnant ambient light. 


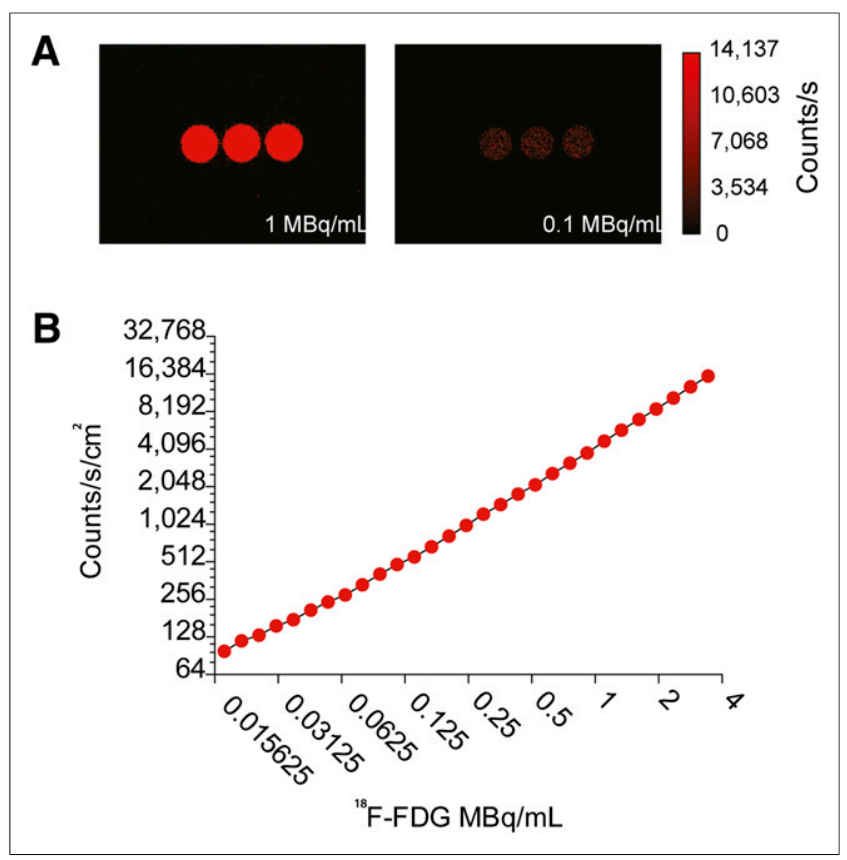

FIGURE 1. Sensitivity and linearity of intensified CCD to detect CL from ${ }^{18} \mathrm{~F}-\mathrm{FDG}$. (A) $\mathrm{CL}$ imaging of multiwell plate with activity at 1 and $0.1 \mathrm{MBq} / \mathrm{mL}$. (B) Linearity analysis of background-subtracted and decay-corrected region of interest of $\mathrm{CL}$ photon flux from wells using intensified CCD with settings optimized for the activity concentrations.

\section{Camera Linearity and Sensitivity}

The camera performance characteristics were evaluated using ${ }^{18}$ F-FDG placed in a 24 -well polycarbonate plate with a black, opaque bottom (Corning Life Sciences). The concentrated activity was dissolved in a $25 \%$ (by weight) solution of $\mathrm{NaCl}$ in water to approximate a refractive index of 1.38 , to give $200 \mu \mathrm{Ci}$ (or approximately 7.2 MBq) per well, in triplicate. The sample was placed $8 \mathrm{~cm}$ from the camera lens and imaged for $5 \mathrm{~min}$. Sequential acquisitions were obtained over time as the activity decayed. A well without activity was used as the background, although with the intensified CCD system, background areas produce negligible signal $(<0.1 \%$ of the signal from a well with activity). A region of interest around this well was subtracted from the region of interest of the three ${ }^{18} \mathrm{~F}-\mathrm{FDG}$-containing wells. Decay-corrected values of the photon flux are presented, normalized by the area of the well $\left(1.91 \mathrm{~cm}^{2}\right)$.

\section{Study Design}

Patients were enrolled under the auspices of a protocol approved by the Memorial Sloan-Kettering Cancer Center Institutional Review Board (protocol 12-050), with ClinicalTrials.gov identifier NCT01664936. The goals of this study were to determine the overall feasibility of acquiring CL emissions from patients undergoing routine diagnostic scans with ${ }^{18} \mathrm{~F}-\mathrm{FDG}$ and to correlate optical imaging with uptake of radiotracer as determined by PET. Head and neck patients scheduled for routine clinical ${ }^{18} \mathrm{~F}$-FDG PET scans were selected for the study and approached. Patients who consented before their scheduled scan and signed a waiver underwent CL imaging after the completion of the PET/CT scan. Four patients have been imaged in the initial phase of this trial. Two scans were completed for each patient, one on the primary site of lymph node uptake and one on the contralateral side of the patient without nodal disease as a negative control. The scan duration was $5 \mathrm{~min}$ per acquisition.

\section{RESULTS}

\section{Camera Performance}

We investigated the sensitivity of the intensified CCD system to detect levels of CL across a wide range of photon flux. For use as a model system, we placed a triplicate source of ${ }^{18} \mathrm{~F}-\mathrm{FDG}$ in a multiwell plate and recorded serial images of the light over the course of decay (Fig.1A). In Figure 1, we have false-colored the light intensity of the CL sources for representative time points acquired at 1 and $0.1 \mathrm{MBq} / \mathrm{mL}$ in each well. The test of linearity of the photon flux measurements revealed a correlation of $r^{2}=$ 0.9991 between activity and flux (Fig. 1B).

\section{Clinical Results}

The pilot study involved 4 patients (2 lymphoma, 1 lung cancer, and 1 breast cancer) who underwent our institution's standard diagnostic ${ }^{18} \mathrm{~F}$-FDG protocol. In addition, 2 patients with lymphoma but normal lymph nodes on PET scanning were imaged. These patients did not show significantly elevated CL signal, and their data are included in Supplemental Figure 1 (supplemental materials are available at http://jnm.snmjournals.org). Lymph nodes in the neck or axilla were imaged after an uptake time of $70 \pm 3$ min after injection of $455 \pm 26$ (SD) MBq of ${ }^{18}$ F-FDG. In a representative patient (58-y-old man with lymphoma) who underwent PET/CT $68 \mathrm{~min}$ after injection of $470 \mathrm{MBq}{ }^{18} \mathrm{~F}-\mathrm{FDG}$ followed by $\mathrm{CL}$ and white-light photographic imaging (Fig. 2), uptake was localized to an enlarged left axillary node (Fig. 2E; maximal uptake, $0.05 \mathrm{MBq} / \mathrm{mL}$ ). Immediately after PET, CL

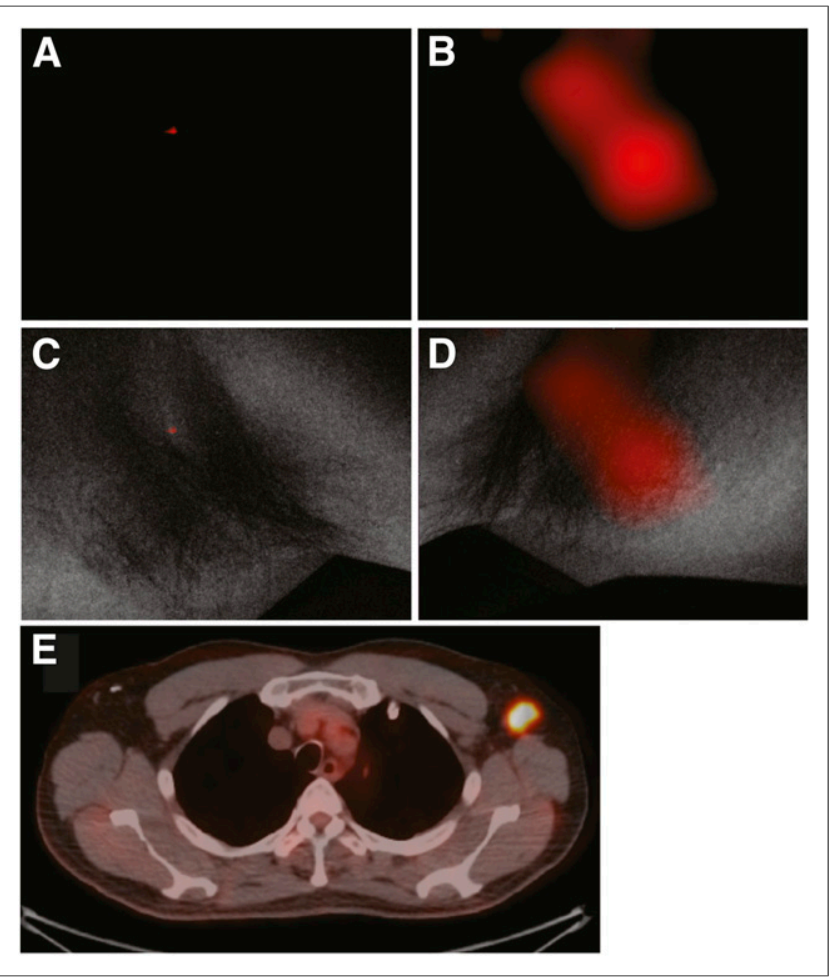

FIGURE 2. Representative $\mathrm{CL}$ and PET/CT images of ${ }^{18} \mathrm{~F}-\mathrm{FDG}-$ positive axillary lymph node. ( $A$ and $B$ ) CL scans of right and left axillae, respectively. (C) Negative $C L$ scan in light-protected environment of right axilla without ${ }^{18} \mathrm{~F}-\mathrm{FDG}$-positive lymph node, overlaid with whitelight photograph. No significant $\mathrm{CL}$ emission from ${ }^{18} \mathrm{~F}-\mathrm{FDG}$ decay is seen. (D) White-light photograph from left axilla, overlaid with significant $C L$ signal (B). (E) This signal colocalized with PET/CT finding. 
images of both axillae were acquired with the high-sensitivity intensified CCD camera over 5 min in the light-restricted environment. $\mathrm{CL}$ and overlay images of the right axilla did not show any significant optical signal over background (Figs. 2A and 2C). In contrast, localized signal is observed at the site of the node in the left axilla (Figs. 1B and 1D). The light that was emitted correlated weakly with the measured activity accumulated in the nodes (Fig. 3; Supplemental Table 1). A less stringent correlation is expected since the Cerenkov light (as any other light) will be absorbed on its way to the surface, depending on the varying thickness and composition of the tissue. The Cerenkov signals originating from the PET-positive sides are however significantly higher than those from the PET-negative control side, which are at noise level $(P=0.02$, Fig. 3B).

\section{DISCUSSION}

CL imaging is a new method for noninvasive optical detection of disease using PET tracers. This technology has demonstrated significant potential for a range of applications by visualizing the optical emissions of a tracer, in addition to PET imaging; for example, for intraoperative guidance in the detection of positive lymph nodes (14).

Legitimate questions concerning the clinical applicability of the use of CL for imaging in humans can be raised given the differences between preclinical studies and patient work, such as the dose given and the depth of the site of interest. Diagnostic nuclear medicine studies in small animals often use a much greater activity per mass ratio than would be permissible in humans. For example, the doses of $11.1 \mathrm{MBq}$, or $300 \mu \mathrm{Ci}$, of ${ }^{18} \mathrm{~F}-$ FDG used in the initial CL imaging rodent work (8) are in line with those commonly used for small-animal PET at many academic institutions. An approximate weight for an 8-wk-old mouse (frequently used in research) is $25 \mathrm{~g}$, resulting in $444 \mathrm{MBq} / \mathrm{kg}$. At Memorial Sloan-Kettering Cancer Center, standard guidelines prescribe $444 \mathrm{MBq}(12 \mathrm{mCi})$ for a $75-\mathrm{kg}$ patient, to yield $5.92 \mathrm{MBq} / \mathrm{kg}$.

A consideration in all optical imaging studies is that the depthpenetration issues are greater in humans than in small animals (scattering and absorption by tissues is many orders of magnitude greater for optical-wavelength radiation than for high-energy photons). Tumor visualization by CL imaging in small-animal models of superficial subcutaneous xenografts can thus be thought of as the ideal setting for ultraviolet- and blue-wavelength Cerenkov radiation. Deeper sites, such as in larger animals and humans, become exponentially more difficult to image because of the absorbing properties of tissue. Thus, this study required the
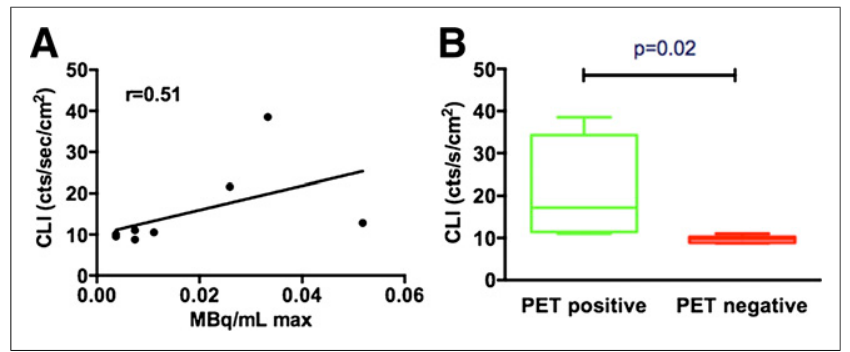

FIGURE 3. (A) Correlation graph between PET (MBq/mL max) and $\mathrm{CL}$ imaging count rate. (B) Box plot comparing Cerenkov emission from pathologic, PET-positive lymph nodes vs. contralateral PET-negative control, demonstrating significantly higher signal from PET-positive side. use of an ultrasensitive photon-detecting device and strict restriction of ambient light.

Despite the technical challenges of detecting CL imaging at the doses administered to patients, the work of Spinelli et al. (12) has shown that imaging in a patient is possible, though they demonstrated clinical CL only in the context of therapeutic doses of the CL-producing radionuclide ${ }^{131}$ I. However, diagnostic imaging makes up the bulwark of the procedures in nuclear medicine. For PET, most such work uses ${ }^{18}$ F-FDG. In this study, CL imaging was achieved in patients undergoing standard diagnostic ${ }^{18} \mathrm{~F}-\mathrm{FDG}$ scanning. We were able to localize optical signal to nodes that displayed uptake above the background level (Fig. 2). The CL signal was detectable in nodes with local activity as low as 0.05 $\mathrm{MBq} / \mathrm{mL}$ (0.03-0.3 MBq/mL; median, $0.05 \mathrm{MBq})$. This level was equivalent to $0.01 \%$ injected dose of ${ }^{18} \mathrm{~F}$-FDG localizing to lymph nodes $1.6 \pm 0.5 \mathrm{~cm}$ under the skin.

To the best of our knowledge, our study was the first to demonstrate the feasibility of optical imaging of CL emissions from diagnostic nuclear scans. This demonstration is significant, because the activity of CL emissions from diagnostic nuclear scans are approximately 4 orders of magnitude less than were the $\mathrm{CL}$ emissions observed from one other patient receiving a therapeutic dose of ${ }^{131}$ I for radioablation of hyperthyroidism (12), with typical uptake of $64 \%$ or greater (15). This characteristic of CL has the advantage of minimizing the activity requirements and subsequent dose for medical personnel in clinical CL imaging. In addition, the results of this pilot study accomplished the primary aim of our ongoing clinical trial in demonstrating a good correlation between PET and CL imaging (Fig. 3).

This work and that of Spinelli et al. (12) have shown that patient imaging is possible by detecting Cerenkov photons. In these initial evaluations of clinical CL imaging we studied a relatively heterogeneous patient population. Although our findings are encouraging, completion of the larger study (NCT01664936) is required to establish the value of CL imaging in the clinic. Advances in camera sensitivity for the detection of these photons in deeper tissue (16), as well as the use of higher-energy positron emitters (which produce more light (17)), can be considered future avenues for development of this technique.

\section{CONCLUSION}

The preliminary results from this clinical trial demonstrate that diagnostic doses of ${ }^{18}$ F-FDG can be imaged using sensitive optical equipment. The CL imaging technique demonstrated uptake in a short time and exhibited a strong correlation with the calculated maximum standarized uptake value from PET. Advances in acquisition technology are expected to increase sensitivity and reduce the duration of the imaging.

\section{DISCLOSURE}

The costs of publication of this article were defrayed in part by the payment of page charges. Therefore, and solely to indicate this fact, this article is hereby marked "advertisement" in accordance with 18 USC section 1734. This work was supported in part by the R25T Molecular Imaging Fellowship "Molecular Imaging Training in Oncology" (5R25CA096945-07), by the NIH (1R01EB01494), and by the Starr Cancer Consortium (I4-A427). No other potential conflict of interest relevant to this article was reported. 


\section{ACKNOWLEDGMENTS}

We thank the radiopharmacy staff at MSKCC, the nurses and administrative staff of the Molecular Imaging and Therapy Service, and the research study assistants.

\section{REFERENCES}

1. Ntziachristos V, Ripoll J, Wang LV, Weissleder R. Looking and listening to light. Nat Biotechnol. 2005;23:313-320.

2. Willmann JK, van Bruggen N, Dinkelborg LM, Gahbhir SS. Molecular imaging in drug development. Nat Rev Drug Discov. 2008;7:591-607.

3. Byrne WL, Delille A, Kuo C, et al. Use of optical imaging to progress novel therapeutics to the clinic. J Control Release. May 13, 2013 [Epub ahead of print].

4. Cho JS, Taschereau R, Olma S, et al. Cerenkov radiation imaging as a method for quantitative measurements of beta particles in a microfluidic chip. Phys Med Biol. 2009;54:6757-6771.

5. Spinelli AE, D'Ambrosio D, Calderan L, Marengo M, Sbarbati A, Boschi F. Cerenkov radiation allows in vivo optical imaging of positron emitting radiotracers. Phys Med Biol. 2010;55:483-495.

6. Robertson R, Germanos MS, Li C, Mitchell GS, Cherry SR, Silva MD. Optical imaging of Cerenkov light generation from positron-emitting radiotracers. Phys Med Biol. 2009;54:N355-N365.

7. Cherenkov PA. Visible emission of clean liquids by action of gamma-radiation. Dokl Akad Nauk SSSR. 1934;2:451-452.
8. Thorek DLJ, Robertson R, Bacchus WA, et al. Cerenkov imaging: a new modality for molecular imaging. Am J Nucl Med Mol Imaging. 2012;2:163-173.

9. Xu Y, Chang E, Liu H, Jiang H, Gambhir SS, Chen Z. Proof-of-concept study of monitoring cancer drug therapy with Cerenkov luminescence imaging. $\mathrm{J} \mathrm{Nucl}$ Med. 2012;53:312-317.

10. Beattie BJ, Thorek DLJ, Schmidtlein CR, Pentlow PS, Humm JL, Hielscher AH. Quantitative modeling of Cerenkov light production efficiency from medical radionuclides. PLOS ONE. 2012;7:e31402.

11. Chin PT, Welling MM, Meskers SC, Valdes Olmos RA, Tanke H, van Leeuwen FW. Optical imaging as an expansion of nuclear medicine. Eur J Nucl Med Mol Imaging. 2013;40:1283-1291.

12. Spinelli AE, Ferdeghini M, Cavedon C, et al. First human Cernekography. J Biomed Opt. 2013;18:20502.

13. Schneider CA, Rasband WS, Eliceiri KW. NIH Image to ImageJ: 25 years of image analysis. Nat Methods. 2012;9:671-675.

14. Thorek DLJ, Abou DS, Beattie BJ, et al. Positron lymphography: multimodal, high-resolution, dynamic mapping and resection of lymph nodes after intradermal injection of ${ }^{18}$ F-FDG. $J$ Nucl Med. 2012;53:1438-1445.

15. Nebesio TD, Siddiqui AR, Pescovitz OH, Eugster EA. Time course to hypothyroidism after fixed-dose radioablation therapy of Graves' disease in children. J Pediatr. 2002;141:99-103.

16. Spinelli AE, Boschi F. Optimizing in vivo small animal Cerenkov luminescence imaging. J Biomed Opt. 2012;17:040506.

17. Ruggiero A, Holland JP, Lewis JS, Grimm J. Cerenkov luminescence imaging of medical isotopes. $J$ Nucl Med. 2010;51:1123-1130. 